\title{
A SIMPLE MODIFICATION OF THE BOX AND COX TRANSFORMATION TO SCALE STABILITY AND INVARIANCE
}

\author{
Toshimitsu Hamasaki*, Tomoyuki Sugimoto ${ }^{\dagger}$ and Seo Young Kim ${ }^{\ddagger}$
}

\begin{abstract}
We consider power-transformations to obtain stability and invariance of measurement scale. The transformation discussed here is the normalized form of the powertransformation originally suggested by Schlesselman (1971). The original suggestion is a simple modification of the Box and Cox transformation to scale invariance for measurement units. In addition to discussion on the scale invariance, we study (i) the behaviors of Jacobian of the transformation and (ii) the effect of the modification on the estimates. Then, we show that the modification of the scale invariance improves the performance of estimates, especially the mean estimate. A simulation study is performed to evaluate numerically performances of the modified transformation, compared with the normalized Box and Cox transformation.
\end{abstract}

\section{Introduction}

When applying standard statistical methods to real data, one often hopes that the data may satisfy the three fundamental assumptions. The assumptions are (i) at least approximate normality of the additive errors, (ii) homoscedasticity of error variance, and (iii) simplicity of structure for the expected value of the response. In order to reduce the departures between data and assumptions, Box and Cox (1964) introduced a method to determine a suitable transformation of the positive variable $x_{i}(>0)(i=1, \ldots, n)$. With an unknown transformation parameter $\lambda$, the Box and Cox transformation is defined by

$$
\varphi_{B C}^{(\lambda)}\left(x_{i}\right)= \begin{cases}\frac{x_{i}^{\lambda}-1}{\lambda}, & \lambda \neq 0, \\ \log x_{i}, & \lambda=0 .\end{cases}
$$

The Box and Cox transformation (1) has been widely used since it was first proposed. However, unfortunately, there are two issues with the definition of the transformation. The first is that the range of the transformed variable is restricted according to whether $\lambda$ is positive or negative. This implies that the transformed variable does not cover the entire range of $(-\infty,+\infty)$ and, hence it is not exactly normally distributed (Hernandez and Johnson, 1980; Goto, Matsubara and Tsuchiya, 1983; Sakia, 1991). Thus, it is known that the original

\footnotetext{
*Department of Biomedical Statistics, Osaka University Graduate School of Medicine, 2-2, Yamadaoka, Suita, Osaka 565-0871, Japan E-mail: hamasakt@medstat.med.osaka-u.ac.jp

${ }^{\dagger}$ Department of Mathematical Sciences, Graduate School of Science and Technology, Hirosaki University, 3, Bunkyocho, Hirosaki, Aomori 036-8561, Japan E-mail: tomoyuki@cc.hirosaki-u.ac.jp

${ }^{\ddagger}$ Statistical Research Institute, Korea National Statistical Office, Government Complex Daejeon, 139 Seonsaro, Seo-gu, Daejeon, Republic of Korea, 302-701 E-mail: smilegong@korea.kr

Key words: Geometric mean; Jacobian; Normalized transformation; Median; Sensitivity
} 
variable $x_{i}$ has the power-normal distribution (Goto et al, 1983; Johnson, Kotz and Balakrishnan, 1994). Consequently, only approximate normality is to be expected. This truncation of the transformed variable is important especially in dealing with univariate data. In a strict sense, the methods that do not account for the truncation cannot be trusted, although they are often close enough to be of practical value.

The second is that the Box and Cox power-transformation is not scale invariant except for $\lambda \neq 0$ (Schlesselman, 1971). If rescaling the data, for example, changing the scale of measurement unit of the distance between two objects from "centimeter" to "meter", has no influence on the estimate of the transformation parameter, but different conclusions on the estimates of the mean parameter may be derived from the original and rescaled data. Also, in regression analysis, if the model does not allow a constant term, the difference in regression parameters between the original and rescaled data becomes serious.

Such difficulties with the definition of the Box and Cox power-transformation come from the range of $x_{i}\left(0<x_{i}<\infty\right)$ and the value of " -1 " in the numerator. The value of " -1 " is for the continuity at $\lambda=0$, but it does not depend on the scale of measurement unit of the variable $x_{i}$. Several modifications have been proposed to overcome such difficulties [please see references in Sakia (1991), Hamasaki and Goto (1996) and Hamasaki et al. (2000)]. In this paper, we consider the normalized form of the transformation suggested by Schlesselman (1971). The original suggestion is the simple modification of the Box and Cox power-transformation to the scale invariance, which is given by

$$
\varphi_{S}^{(\lambda)}\left(x_{i}\right)= \begin{cases}\frac{x_{i}^{\lambda}-x_{0}^{\lambda}}{\lambda}, & \lambda \neq 0, \\ \log \left(x_{i} / x_{0}\right), & \lambda=0,\end{cases}
$$

where $x_{0}$ is an arbitrary positive constant in the scale of measurement unit of the variable $x$. Schlesselman (1971) suggests the use of a given value on the scales of $x$ as the value of $x_{0}$, such as average $x_{A}$, geometric mean $x_{G}$ and median $x_{M}$. Then, the normalized transformation of (2) is given by

$$
\phi_{S}^{(\lambda)}\left(x_{i}\right)=\frac{\varphi_{S}^{(\lambda)}\left(x_{i}\right)}{J_{\varphi_{S}}^{1 / n}(\lambda)}= \begin{cases}\frac{x_{i}^{\lambda}-x_{0}^{\lambda}}{\lambda x_{G}^{\lambda-1}}, & \lambda \neq 0, \\ x_{G} \log \left(x_{i} / x_{0}\right), & \lambda=0\end{cases}
$$

where $J_{\varphi_{S}}$ is the Jacobian of the transformation $x_{i} \rightarrow \varphi_{S}^{(\lambda)}\left(x_{i}\right)$, which is given by

$$
J_{\varphi_{S}}(\lambda)= \begin{cases}x_{G}^{n(\lambda-1)} & \lambda \neq 0 \\ x_{G}^{n}, & \lambda=0 .\end{cases}
$$

In analysis of data, it may be often preferable to use the normalized transformation by the $n$-th root of the Jacobian of a transformation. This is because this normalization may remove the $\lambda$-dependent scale effect (Hinkley and Runger, 1984) and then scale dependent statistics can be directly compared between the original and transformed data. Thus, the normalized transformation of (1) is given by

$$
\phi_{B C}^{(\lambda)}\left(x_{i}\right)=\frac{\varphi_{B C}^{(\lambda)}\left(x_{i}\right)}{J_{\varphi_{B C}}^{1 / n}(\lambda)}= \begin{cases}\frac{x_{i}^{\lambda}-1}{\lambda x_{G}^{\lambda-1},} & \lambda \neq 0, \\ x_{G} \log x_{i}, & \lambda=0,\end{cases}
$$


where $J_{\varphi_{B C}}(\lambda)$ is the Jacobian of the transformation $x_{i} \rightarrow \varphi_{B C}^{(\lambda)}\left(x_{i}\right)$ and $J_{\varphi_{B C}}(\lambda)=J_{\varphi_{S}}(\lambda)$. The two transformations (1) and (2) have the same form of Jacobian. In addition, the Jacobians of the transformations (3) and (5) are given by

$$
J_{\phi_{B C}}(\lambda)= \begin{cases}1+\frac{1-\lambda}{n \lambda} \sum_{i=1}^{n}\left(1-x_{i}^{-\lambda}\right), & \lambda \neq 0, \\ 1+\log x_{G}, & \lambda=0,\end{cases}
$$

and

$$
J_{\phi_{S}}(\lambda)= \begin{cases}1+\frac{1-\lambda}{n \lambda} \sum_{i=1}^{n}\left[1-\left(\frac{x_{i}}{x_{0}}\right)^{-\lambda}\right], & \lambda \neq 0, \\ 1+\log x_{G}-\log x_{0}, & \lambda=0\end{cases}
$$

respectively. Unfortunately, as seen in definitions of the transformations (2), (3) and (5), their transformed values do not cover the entire range of $(-\infty, \infty)$.

In this paper, we discuss the properties, performances and practical applicability of the normalized transformation (3) compared with the normalized transformation of (5) in the analysis of univariate data. We investigate the stability and invariance of measurement scale, and their effect on the estimates. Then we show that the modification of the scale invariance improves the performance of estimates, especially the mean estimate. The modification is an origin shift so that it should not be relevant in regression analysis if the model includes a constant term. However, it would be quite important in estimating means in the analysis of univariate data.

\section{Invariance and stability of measurement scale of the modified transformation}

\subsection{Invariance of measurement scale}

We here consider the rescaling of $x_{i} \stackrel{a}{\longrightarrow} a x_{i}$, where $a$ is a positive constant. Following the procedure in Schlesselman (1971), under the rescaling of $x_{i} \stackrel{a}{\longrightarrow} a x_{i}$, we can see from (1) and (2) that, for $x_{0} \neq 1$,

$$
\varphi_{B C}^{(\lambda)}\left(x_{i}\right) \stackrel{a}{\longrightarrow} a^{\lambda} \varphi_{B C}^{(\lambda)}\left(x_{i}\right)+\left(a^{\lambda}-1\right) / \lambda \quad \text { and } \quad \varphi_{S}^{(\lambda)}\left(x_{i}\right) \stackrel{a}{\longrightarrow} a^{\lambda} \varphi_{S}^{(\lambda)}\left(x_{i}\right),
$$

respectively. Therefore, the transformation (1) is not scale invariant for $\lambda \neq 0$, but the transformation (2) is scale invariant for all values of $\lambda$. Similarly, under the rescaling of $x_{i} \stackrel{a}{\longrightarrow} a x_{i}$, we can see from the transformations (5) and (3) that, for $x_{0} \neq 1$,

$$
\phi_{B C}^{(\lambda)}\left(x_{i}\right) \stackrel{a}{\longrightarrow} a^{\lambda} \phi_{B C}^{(\lambda)}\left(x_{i}\right)+\left(a^{\lambda}-1\right) /\left(\lambda x_{G}^{\lambda-1}\right) \quad \text { and } \quad \phi_{S}^{(\lambda)}\left(x_{i}\right) \stackrel{a}{\longrightarrow} a^{\lambda} \phi_{S}^{(\lambda)}\left(x_{i}\right),
$$

respectively. Therefore, the transformation (5) is not scale invariant for $\lambda \neq 0$, but the transformation (3) is scale invariant for all values of $\lambda$.

\subsection{Sensitivity of the mean and variance estimates}

The transformations (3) and (5) involve changes of origin and scale, both of which depend on $\lambda$. We now consider the degree of how much the $\lambda$-scale effect can be removed by the normalization. Then, one suitable measure of the scale change is the $n$-th root of the Jacobian of the transformation (Hinkley and Runger, 1984). From (6) and (7), the average scale factor $J_{\phi_{B C}}^{1 / n}(\lambda)$ and $J_{\phi_{S}}^{1 / n}(\lambda)$ should be approximately one for a large $n$. If $x_{i}^{\lambda}$ is a 
normal variable with mean $\lambda \mu_{\varphi_{B C}}+1$ and varianec $\sigma_{\varphi_{B C}}^{2}$, for $\lambda \neq 0$, the application of a Taylor expansion provides the first order approximation

$$
\mathrm{E}\left[J_{\phi_{B C}}(\lambda)\right] \approx 1+\frac{\lambda-1}{\lambda}\left(1-\left(\lambda \mu_{\varphi_{B C}}+1\right)^{-1}\left[1+\frac{G^{2-2 \lambda} \sigma_{\varphi_{B C}}^{2}}{2\left(\lambda \mu_{\varphi_{B C}}+1\right)^{2}}\right]\right),
$$

where $G=\exp \mathrm{E}[\log x]$ is the limit of the geometric mean $x_{G}$. Similarly, we have the first order approximation

$$
\mathrm{E}\left[J_{\phi_{S}}(\lambda)\right] \approx 1+\frac{\lambda-1}{\lambda}\left(1-x_{0}^{\lambda}\left(\lambda \mu_{\varphi_{B C}}+1\right)^{-1}\left[1+\frac{G^{2-2 \lambda} \sigma_{\varphi_{B C}}^{2}}{2\left(\lambda \mu \varphi_{y_{B C}}+1\right)^{2}}\right]\right)
$$

if $x_{0}=\left(\lambda \mu_{\varphi_{B C}}+1\right)^{1 / \lambda}$, then we have

$$
\mathrm{E}\left[J_{\phi_{S}}(\lambda)\right] \approx 1-\frac{\lambda-1}{\lambda} \frac{G^{2-2 \lambda} \sigma_{\varphi_{B C}}^{2}}{2\left(\lambda \mu_{\varphi_{B C}}+1\right)^{2}}
$$

From the comparison of (8) and (9), if $x_{0}=\left(\lambda \mu_{\varphi_{B C}}+1\right)^{1 / \lambda}$, it is clear that $J_{\phi_{S}}^{1 / n}(\lambda)$ tends more quickly to one than $J_{\phi_{B C}}^{1 / n}(\lambda)$ and therefore the stability of measurement scale in the transformation (3) is better than the transformation (5). Thus, the appropriate value of $x_{0}$ for a stable measurement scale depends on $\lambda$. For example, for $\lambda=0$, the geometric mean $x_{G}$ is clearly appropriate as the value of $x_{0}$. Then, the median in the original scale $x_{M}$ is approximately given by $\left(\lambda \mu_{\varphi_{B C}}+1\right)^{1 / \lambda}$ if the truncation of the transformed variable can be small enough to be ignored (Goto et al., 1983). Therefore, the median on the original scale may be recommended for a stable measurement scale.

\section{Maximum likelihood estimates of the modified transformations}

\subsection{Comparison of forms for maximum likelihood estimates}

In the Box and Cox procedure, the transformed variable is assumed to be approximately normally distributed with mean $\mu_{\varphi_{B C}}$ and variance $\sigma_{\varphi_{B C}}^{2}$. We here assume that $x_{i}$ is large enough so that no restriction on $\lambda$ described above is necessary. Apart from a constant, for $\lambda \neq 0$, the log-likelihood is give by

$$
L_{\varphi_{B C}}=-\frac{1}{2} n \log \sigma_{\varphi_{B C}}^{2}-\frac{1}{2 \sigma_{\varphi_{B C}}^{2}} \sum_{i=1}^{n}\left(\varphi_{B C}^{(\lambda)}\left(x_{i}\right)-\mu_{\varphi_{B C}}\right)^{2}+n(\lambda-1) \log x_{G} .
$$

In practice, however, the estimates of the parameters are found in two stages. First, for fixed $\lambda$, the $\log$-likelihood (10) is maximized with respect to $\mu_{\varphi_{B C}}$ and $\sigma_{\varphi_{B C}}^{2}$. Then the $\log$ likelihood (10) for fixed $\lambda$ is the log-likelihood for the least-squares problem with $\varphi_{B C}^{(\lambda)}\left(x_{i}\right)$. Therefore, if we denote the maximum likelihood estimates of $\mu_{\varphi_{B C}}$ and $\sigma_{\varphi_{B C}}^{2}$ for the fixed $\lambda$ by $\hat{\mu}_{\varphi_{B C}}(\lambda)$ and $\hat{\sigma}_{\varphi_{B C}}^{2}(\lambda)$, we have

$$
\hat{\mu}_{\varphi_{B C}}(\lambda)=\frac{\sum_{i=1}^{n} \varphi_{B C}^{(\lambda)}\left(x_{i}\right)}{n} \quad \text { and } \quad \hat{\sigma}_{\varphi_{B C}}^{2}(\lambda)=\frac{\sum_{i=1}^{n}\left(\varphi_{B C}^{(\lambda)}\left(x_{i}\right)-\hat{\mu}_{\varphi_{B C}}(\lambda)\right)^{2}}{n} .
$$

Then substitution of $\hat{\mu}_{\varphi_{B C}}(\lambda)$ and $\hat{\sigma}_{\varphi_{B C}}^{2}(\lambda)$ into the log-likelihood (10) yields, apart from a constant, the maximized log-likelihood 


$$
L_{\varphi_{B C} \max }(\lambda)=-\frac{1}{2} n \log \hat{\sigma}_{\varphi_{B C}}^{2}(\lambda)+n(\lambda-1) \log x_{G}
$$

Secondly, the log-likelihood (11) is maximized with respect $\lambda$ to find the maximum likelihood estimates $\hat{\lambda}$ of $\lambda$. Consequently, we have the maximum likelihood estimates $\hat{\lambda}, \hat{\mu}_{\varphi_{B C}}(\hat{\lambda})$ and $\hat{\sigma}_{\varphi_{B C}}^{2}(\hat{\lambda})$.

Similarly, if the transformed variable $\varphi_{S}^{(\lambda)}\left(x_{i}\right)$ is assumed to be approximately normally distributed with mean $\mu_{\varphi_{S}}$ and common variance $\sigma_{\varphi_{S}}^{2}$, the log-likelihood is given by

$$
L_{\varphi_{S}}=-\frac{1}{2} n \log \sigma_{\varphi_{S}}^{2}-\frac{1}{2 \sigma_{\varphi_{S}}^{2}} \sum_{i=1}^{n}\left(\varphi_{S}^{(\lambda)}\left(x_{i}\right)-\mu_{\varphi_{S}}\right)^{2}+n(\lambda-1) \log x_{G}
$$

Then, since $\varphi_{S}^{(\lambda)}\left(x_{i}\right)$ can be rewritten as $\varphi_{S}^{(\lambda)}\left(x_{i}\right)=\varphi_{B C}^{(\lambda)}\left(x_{i}\right)-\left(x_{0}^{\lambda}-1\right) / \lambda$ and the origin shift has no effect on the estimate of variance, we have $\hat{\mu}_{\varphi_{S}}(\lambda)=\hat{\mu}_{\varphi_{B C}}(\lambda)-\left(x_{0}^{\lambda}-1\right) / \lambda$ and $\hat{\sigma}_{\varphi_{S}}^{2}(\lambda)=\hat{\sigma}_{\varphi_{B C}}^{2}(\lambda)$. Hence, $L_{\varphi_{S} \max }(\lambda)=L_{\varphi_{B C} \max }(\lambda)$. From these results, except for $\mu$, the same estimates of $\lambda$ and $\sigma^{2}$ are found in the transformations (1) and (2).

Next we consider the transformations (3) and (5) in the same way. For $\lambda \neq 0$, the log-likelihoods for $\phi_{B C}^{(\lambda)}\left(x_{i}\right)$ and $\phi_{Z}^{(\lambda)}\left(x_{i}\right)$ are given by

$$
L_{\phi_{B C}}=-\frac{1}{2} n \log \sigma_{\phi_{B C}}^{2}-\frac{1}{2 \sigma_{\phi_{B C}}^{2}} \sum_{i=1}^{n}\left(\phi_{B C}^{(\lambda)}\left(x_{i}\right)-\mu_{\phi_{B C}}\right)^{2}+\log J_{\phi_{B C}}(\lambda)
$$

and

$$
L_{\phi_{S}}=-\frac{1}{2} n \log \sigma_{\phi_{S}}^{2}-\frac{1}{2 \sigma_{\phi_{S}}^{2}} \sum_{i=1}^{n}\left(\phi_{S}^{(\lambda)}\left(x_{i}\right)-\mu_{\phi_{S}}\right)^{2}+\log J_{\phi_{S}}(\lambda)
$$

Since $\phi_{S}^{(\lambda)}\left(x_{i}\right)$ can be rewritten as $\phi_{S}^{(\lambda)}\left(x_{i}\right)=\phi_{B C}^{(\lambda)}\left(x_{i}\right)-\left(x_{0}^{\lambda}-1\right) /\left(\lambda x_{G}^{\lambda-1}\right)$ and then $\hat{\sigma}_{\phi_{S}}^{2}(\lambda)=$ $\hat{\sigma}_{\phi_{B C}}^{2}(\lambda)$, the difference between the maximized log-likelihoods $L_{\phi_{S} \max }(\lambda)$ and $L_{\phi_{B C} \max }(\lambda)$ leads to the difference between the Jacobians.

\subsection{Sensitivity of the mean and variance estimates to change in transformation}

In this section, we discuss the sensitivity of the mean and variance estimates to change in $\lambda$. Then, similarly as in Duan (1993) and Hamasaki and Goto (1996) in order to measure the sensitivity, we use the following two quantities:

$$
R(\hat{\mu}(\lambda))=\lim _{\Delta \rightarrow 0} \frac{(\hat{\mu}(\lambda+\Delta)-\hat{\mu}(\lambda)) / \Delta}{\hat{\mu}(\lambda)}=\frac{\hat{\mu}^{\prime}(\lambda)}{\hat{\mu}(\lambda)}
$$

and

$$
R\left(\hat{\sigma}^{2}(\lambda)\right)=\lim _{\Delta \rightarrow 0} \frac{\left(\hat{\sigma}^{2}(\lambda+\Delta)-\hat{\sigma}^{2}(\lambda)\right) / \Delta}{\hat{\sigma}^{2}(\lambda)}=\frac{\hat{\sigma}^{2 \prime}(\lambda)}{\hat{\sigma}^{2}(\lambda)}
$$

where $\Delta$ is the given perturbation, and $\hat{\mu}^{\prime}(\lambda)$ and $\sigma^{2 \prime}(\lambda)$ are the derivatives of the mean and variance with respect to $\lambda$.

First we consider the sensitivity of $\hat{\mu}_{\phi_{B C}}(\lambda)$ and $\hat{\mu}_{\phi_{S}}(\lambda)$ to change in $\lambda$. Under the regularity conditions for convergence of $\hat{\lambda}$ to the true transformation $\lambda_{0}$, for a large $n$, we have 


$$
\mathrm{E}\left[\hat{\mu}_{\phi_{B C}}\left(\lambda_{0}\right)\right] \approx \frac{\mu_{\varphi_{B C}}}{G^{\lambda_{0}-1}}
$$

and

$$
\mathrm{E}\left[\hat{\mu}_{\phi_{S}}\left(\lambda_{0}\right)\right] \approx \frac{\mu_{\varphi_{B C}}}{G^{\lambda_{0}-1}}-\frac{x_{0}^{\lambda_{0}}-1}{\lambda_{0} G^{\lambda_{0}-1}}
$$

In addition, if we denote the derivatives of the means $\hat{\mu}_{\phi_{B C}}\left(\lambda_{0}\right)$ and $\hat{\mu}_{\phi_{S}}\left(\lambda_{0}\right)$ by $\hat{\mu}_{\phi_{B C}}^{\prime}\left(\lambda_{0}\right)$ and $\hat{\mu}_{\phi_{S}}^{\prime}\left(\lambda_{0}\right)$, we have

$$
\mathrm{E}\left[\hat{\mu}_{\phi_{B C}}^{\prime}\left(\lambda_{0}\right)\right] \approx \frac{\mathrm{E}\left[x^{\lambda_{0}} \log x\right]}{G^{\lambda_{0}-1}}-\frac{c \mu_{\varphi_{B C}}}{G^{\lambda_{0}-1}}
$$

and

$$
\mathrm{E}\left[\hat{\mu}_{\phi_{S}}^{\prime}\left(\lambda_{0}\right)\right] \approx \frac{\mathrm{E}\left[x^{\lambda_{0}} \log x\right]}{\lambda_{0} G^{\lambda_{0}-1}}-\frac{c \mu_{\varphi_{B C}}}{G^{\lambda_{0}-1}}-\frac{x_{0}^{\lambda_{0}} \log x_{0}}{\lambda_{0} G^{\lambda_{0}-1}}+\frac{c\left(x_{0}^{\lambda_{0}}-1\right)}{\lambda_{0} G^{\lambda_{0}-1}},
$$

where $c=1 / \lambda_{0}+\log G$. If $x_{i}^{\lambda}$ is a normal variable with mean $\lambda_{0} \mu_{\varphi_{B C}}+1$ from the result in Hinkley (1989), then we have the approximation, for a large $\mu_{\varphi_{B C}}$,

$$
\mathrm{E}\left[x^{\lambda_{0}} \log x\right] \approx \frac{\left(\lambda_{0} \mu_{\varphi_{B C}}+1\right) \log \left(\lambda_{0} \mu_{\varphi_{B C}}+1\right)}{\lambda_{0}}
$$

By the substitution of this approximation (16) into (15), it follows readily that $\mathrm{E}\left[\hat{\mu}_{\phi_{S}}\left(\lambda_{0}\right)\right] \approx$ 0 and $\mathrm{E}\left[\hat{\mu}_{\phi_{S}}^{\prime}\left(\lambda_{0}\right)\right] \approx 0$ if $x_{0}$ is $\left(\lambda_{0} \mu_{\varphi_{B C}}+1\right)^{1 / \lambda_{0}}$. Thus, as described above, the median in the original scale $x_{M}$ is approximately $\left(\lambda_{0} \mu_{\varphi_{B C}}+1\right)^{1 / \lambda_{0}}$ if the truncation of the transformed variable is small enough to be ignored. Therefore, for the transformation (3), since $\mathrm{E}\left[R\left(\hat{\mu}_{\phi_{S}}\left(\lambda_{0}\right)\right)\right] \approx 1, \hat{\mu}_{\phi_{S}}\left(\lambda_{0}\right)$ are stable to change in $\lambda$ if $x_{M}$ is used as the value of $x_{0}$. On the other hand, for the transformation (5), from (12) and (14), we have

$$
\mathrm{E}\left[R\left(\hat{\mu}_{\phi_{B C}}\left(\lambda_{0}\right)\right)\right] \approx \frac{\mathrm{E}\left[x^{\lambda_{0}} \log x\right]}{\mu_{\varphi_{B C}}}-c \approx \mathrm{E}\left[R\left(\hat{\mu}_{\varphi_{B C}}\left(\lambda_{0}\right)\right)\right]-\log G .
$$

Therefore, the value of $R\left(\mu_{\varphi_{B C}}\right)$ depends on $\lambda_{0}$ and is not constant. The substitution of the approximation (16) and $\log G \approx \log \left(\lambda_{0} \mu_{\varphi_{B C}}+1\right) / \lambda_{0}$ into (17) yields

$$
\mathrm{E}\left[R\left(\hat{\mu}_{\phi_{B C}}\left(\lambda_{0}\right)\right)\right] \approx \frac{\left(\lambda_{0} \mu_{\varphi_{B C}}+1-\mu_{\varphi_{B C}}\right) \log \left(\lambda_{0} \mu_{\varphi_{B C}}+1\right)}{\lambda_{0} \mu_{\varphi_{B C}}}-\frac{1}{\lambda_{0}} .
$$

From this result, it is clear that, even if $\mu_{\varphi_{B C}}$ is large, the term related to $\lambda_{0}$ remains and therefore $\hat{\mu}_{\phi_{B C}}\left(\lambda_{0}\right)$ is sensitive to change in $\lambda$.

Next, we consider the estimates of variance $\sigma_{\phi_{B C}}^{2}(\lambda)$ and $\sigma_{\phi_{S}}^{2}(\lambda)$. As we saw in the previous section that $\hat{\sigma}_{\phi_{B C}}^{2}(\lambda)=\hat{\sigma}_{\phi_{S}}^{2}(\lambda)$, we here discuss $\sigma_{\phi_{B C}}^{2}(\lambda)$. Under the regularity conditions for convergence of $\hat{\lambda}$ to the true transformation $\lambda_{0}$, for a large $n$, we have

$$
\mathrm{E}\left[\hat{\sigma}_{\phi_{B C}}^{2}\left(\lambda_{0}\right)\right] \approx \frac{\sigma_{\varphi_{B C}}^{2}}{G^{2 \lambda_{0}-2}}
$$


Furthermore, if we denote the derivative of $\hat{\sigma}_{\phi_{B C}}^{2}\left(\lambda_{0}\right)$ by $\hat{\sigma}_{\phi_{B C}}^{2 \prime}\left(\lambda_{0}\right)$, we have

$$
\mathrm{E}\left[\hat{\sigma}_{\phi_{B C}}^{2 \prime}\left(\lambda_{0}\right)\right] \approx \frac{2 \mathrm{E}\left[x^{\lambda_{0}}\left[x^{\lambda_{0}}-\left(1+\lambda_{0} \mu_{\varphi_{B C}}\right)\right] \log x\right]}{\lambda_{0}^{2} G^{2 \lambda_{0}-2}}-\frac{2\left(1+\lambda_{0} \log G\right) \sigma_{\varphi_{B C}}^{2}}{\lambda_{0} G^{2 \lambda_{0}-2}}
$$

so that

$$
\mathrm{E}\left[R\left(\hat{\sigma}_{\phi_{B C}}^{2}\left(\lambda_{0}\right)\right)\right] \approx \mathrm{E}\left[R\left(\hat{\sigma}_{\varphi_{B C}}^{2}\left(\lambda_{0}\right)\right)\right]-2 \log G .
$$

If $x_{i}^{\lambda}$ is a normal variable with mean $\lambda_{0} \mu_{\varphi_{B C}}+1$ and variance $\sigma_{\varphi_{B C}}^{2}$, using the result in Hinkley (1989), for a large $\mu_{\varphi_{B C}}$, we have the approximation

$$
\mathrm{E}\left[x^{\lambda_{0}}\left[x^{\lambda_{0}}-\left(1+\lambda_{0} \mu_{\varphi_{B C}}\right)\right] \log x\right] \approx \lambda_{0} \sigma_{\varphi_{B C}}^{2}\left[\log \left(\lambda_{0} \mu_{\varphi_{B C}}+1\right)+1\right]
$$

The substitution of the approximation (19) into (18) yields

$$
\mathrm{E}\left[\hat{\sigma}_{\phi_{B C}}^{2 \prime}\left(\lambda_{0}\right)\right] \approx 0
$$

and then $\mathrm{E}\left[R\left(\hat{\sigma}_{\phi_{B C}}^{2}\left(\lambda_{0}\right)\right)\right] \approx 0$. Therefore, for the transformations $(3)$ and $(5)$, both $\hat{\sigma}_{\phi_{B C}}^{2}\left(\lambda_{0}\right)$ and $\hat{\sigma}_{\phi_{S}}^{2}\left(\lambda_{0}\right)$ are stable to change in $\lambda$.

\section{A simulation study}

\subsection{Setting for simulation}

In this section, we carry out a simulation study to evaluate the performances of the normalized transformation (3) compared with the transformation (5).

Data are generated from the power-normal distribution, using the inverse CDF technique (Akima, 1970; Guerra, Tapia and Thompson, 1976). It is well-known that the powernormal distribution has the six typical shapes of the distribution with the shape parameter $\lambda^{*}$ : the L-shaped distribution $\left(\lambda^{*}<0\right)$, the exponential-shaped distribution (Type I) $\left(0<\lambda^{*}<t_{k}\right)$, the exponential-shaped distribution (Type II), the distribution with maximum and minimum $\left(t_{k}<\lambda^{*}<1\right)$, the truncated normal distribution $\left(\lambda^{*}=1\right)$ and the J-shaped distribution $\left(\lambda^{*}>1\right)$, where $t_{k}=4 /\left(k^{2}+4\right)$ and $k$ denotes the standardized truncation point of the transformed variable which is given by $|k|=\left(\lambda^{*} \mu_{\varphi_{B C}}+1\right) /\left(\lambda \sigma_{\varphi_{B C}}\right)$. Therefore, if the coefficient of variation $(\mathrm{CV})$ for the transformed variable is denoted by $\tau=\sigma_{\varphi_{B C}} / \mu_{\varphi_{B C}}$, the mean and variance for the transformed variable $\varphi_{B C}^{(\lambda)}\left(x_{i}\right)$ are given by

$$
\mu_{\varphi_{B C}}=\frac{1}{\lambda^{*}(k \tau-1)} \quad \text { and } \quad \sigma_{\varphi_{B C}}^{2}=\left(\mu_{\varphi_{B C}} \tau\right)^{2} .
$$

For more details of the power-normal distribution, please see Goto et al. (1983)

In this paper, we consider the situation where the truncation is small enough to be ignored and then set $k=5$. This value corresponds to the truncation probability of $0.00003 \%$. Also, since $t_{k}=0.1379$, we set the values of $\lambda$ and $\tau$ as follows: $\lambda^{*}=-1.0,0.10,0.1379,0.5,1.0$ and 2.0, and $\tau=0.1,1.0$ and 2.0. For given values of $\lambda^{*}, k$ and $\tau$, the values of $\mu_{\varphi_{B C}}$ and $\sigma_{\varphi_{B C}}^{2}$ can be determined by (20). For each setting of combinations of parameter values, 10,000 data sets are generated, each with sample sizes of $n=100$ and 200 . The number of replications of 10,000 is expected to provide a $95 \%$ assurance that the error in the estimated mean is less than at least $2.0 \%$ of the standard deviation (SD). In addition, we use the following three values of $x_{0}$ : median $x_{M}$, geometric mean $x_{G}$ and average $x_{A}$. The parameter estimation is carried out for the normalized transformations (3) and (5). 
HAMASAKI, SUGIMOTO and KIM

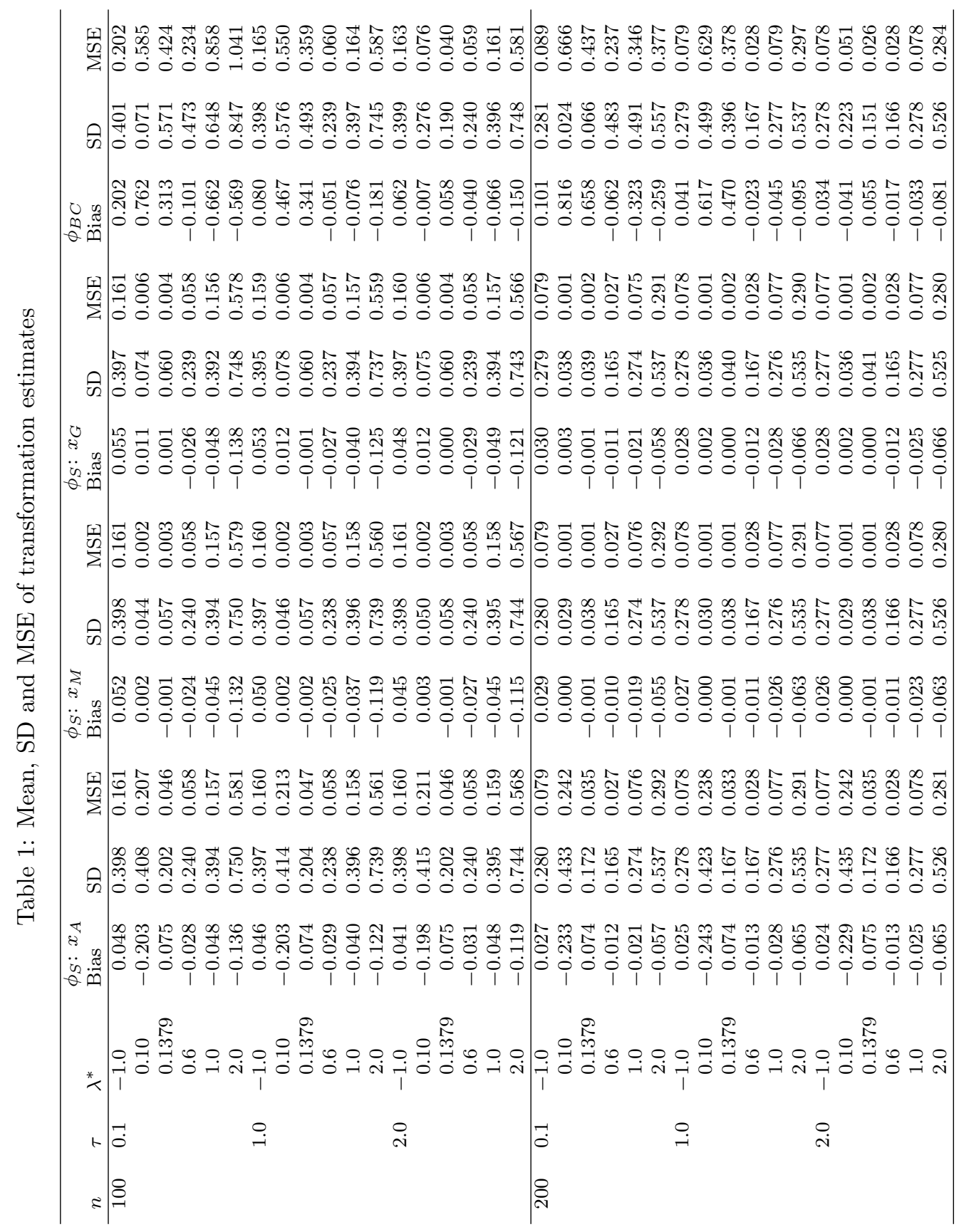




\subsection{Simulation results}

To evaluate the performances of the transformations (3) and (5), we consider the bias and mean square error (MSE) of the estimates from the normalized transformations (3) and (5), and then compute the mean, SD and MSE of the estimates for 10,000 data sets of each setting. Then, in order to compare directly the result of the mean and variance, we convert the estimates of the means $\hat{\mu}_{\phi_{B C}}(\hat{\lambda})$ and $\hat{\mu}_{\phi_{S}}(\hat{\lambda})$ to $\hat{\mu}_{\varphi_{B C}}=x_{G}^{\hat{\lambda}-1} \hat{\mu}_{\phi_{B S}}(\hat{\lambda})$ and $\hat{\mu}_{\varphi_{B C}}=x_{G}^{\hat{\lambda}-1}\left[\hat{\mu}_{\phi_{S}}(\hat{\lambda})+\left(x_{0}^{\hat{\lambda}}-1\right) /\left(\hat{\lambda} x_{G}^{\hat{\lambda}-1}\right)\right]$, respectively, and the estimates of variances $\hat{\sigma}_{\phi_{S}}^{2}(\hat{\lambda})$ and $\hat{\sigma}_{\phi_{B C}}^{2}(\hat{\lambda})$ to $\hat{\sigma}_{\varphi_{B C}}^{2}=x_{G}^{2 \hat{\lambda}-2} \sigma_{\phi_{S}}^{2}(\hat{\lambda})=x_{G}^{2 \hat{\lambda}-2} \sigma_{\phi_{B C}}^{2}(\hat{\lambda})$, respectively. Also, we compute the $n$-th root of Jacobian to evaluate the stability of the transformed scale. Furthermore, we compute the sensitivities $R(\hat{\mu}(\hat{\lambda}))$ and $R\left(\hat{\sigma}^{2}(\hat{\lambda})\right)$ for 10,000 data sets of each configuration to evaluate the sensitivity of the mean and variance to change in $\lambda$. However, here we will just discuss the bias and MSE of transformation estiamte, the stability of the transformed scale and the sensitivity of the mean and variance to change in $\lambda$.

Table 1 provides the bias, SD and MSE of $\hat{\lambda}$ for the transformations (3) and (5). The transformations (3) with $x_{A}, x_{M}$ and $x_{G}$ show no significant bias and MSE of $\hat{\lambda}$ and provide consistently smaller bias and MSE compared with the transformation (5) in all settings. Especially, the transformations (3) with $x_{M}$ provides the smallest values of bias and MSEs of $\hat{\lambda}$. However, the difference is relatively small and seems not to be serious in practical use. In addition, the bias and MSE from the transformations (3) with $x_{A}, x_{M}$ and $x_{G}$ go smaller as the value of $n$ is larger though the bias and MSE do not change with the values of $\lambda^{*}$ and $\tau$ within the same sample size.

Figure 1 provide the box and whisker plot of the $n$-th roots of the Jacobians for the transformations (3) and (5). Except for $\lambda^{*}=0.1$ and 0.1379 , the transformations (3) with $x_{A}, x_{M}$ and $x_{G}$ show the values near one. Also, they generally go better than the transformation (5). In addition, Figures 2 and 3 provide the box and whisker plots of $R(\hat{\mu}(\hat{\lambda}))$ and $R\left(\hat{\sigma}^{2}(\hat{\lambda})\right)$ of mean and variance estimates for the transformations $(3)$ and $(5)$. Except for $\lambda=0.1$ and 0.1379 , the transformations (3) show values near one for $R(\hat{\mu}(\hat{\lambda}))$ and values near zero for $R\left(\hat{\sigma}^{2}(\hat{\lambda})\right)$ when the value of $\tau$ are small and the value of $n$ is large. Also, they generally go better than the transformation (5).

\section{Concluding remarks}

The normalization of a transformation by using the $n$-th root of its Jacobian lies in the center of discussions among Bickel and Doksum (1981), Box and Cox (1982) and Hinkley and Runger (1984) on parameter estimation of the Box and Cox power-transformation model. One of the key issues in the discussions is the sensitivity of the parameter estimates to change in the transformation parameter (Duan, 1991).

In this paper, we consider the normalized form of the power-transformation suggested by Schlesselman (1971). The original suggestion is a simple modification of the Box and Cox transformation, where the value of " -1 " in the numerator for the Box and Cox transformation is replaced by a constant in the scale of measurement units of the original variable in order to retain scale invariance for measurement units. We study the behaviors of Jacobians of the normalized transformations and the effect of modifications on the estimates. The result shows that the normalized transformations can provide a more stable measurement scale compared with the normalized Box and Cox transformation as well as being scale invariant for measurement units. Also, the normalized transformation can practically remove the effect of the transformation-dependent scale on the estimates of the mean and 

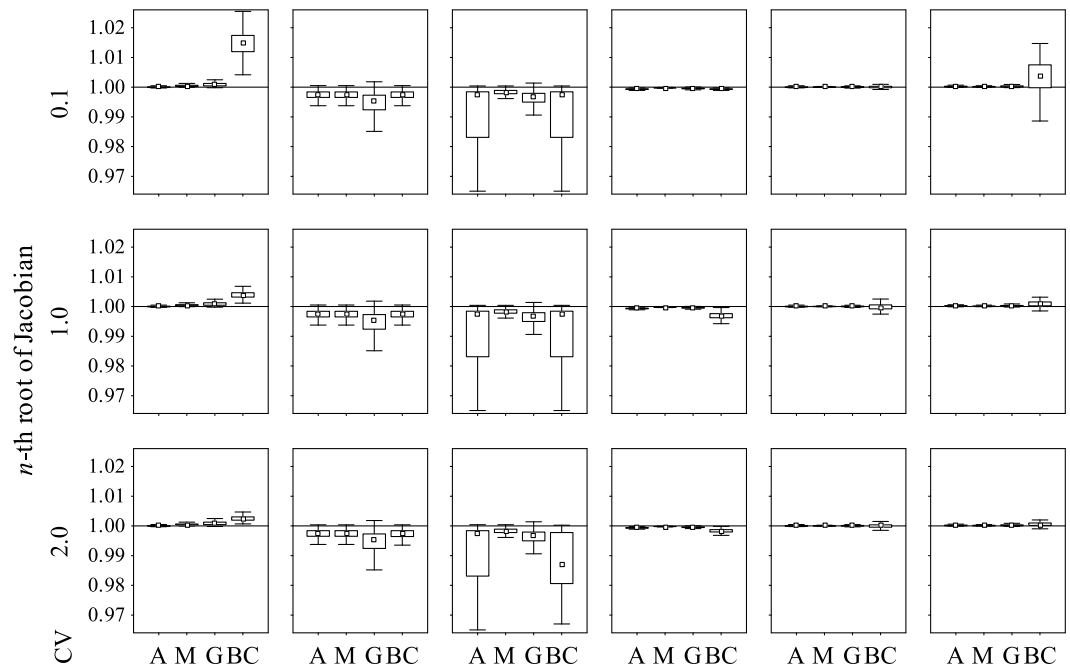

$-1.0$

0.1

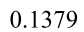

0.6

1.0

Shape Parameter
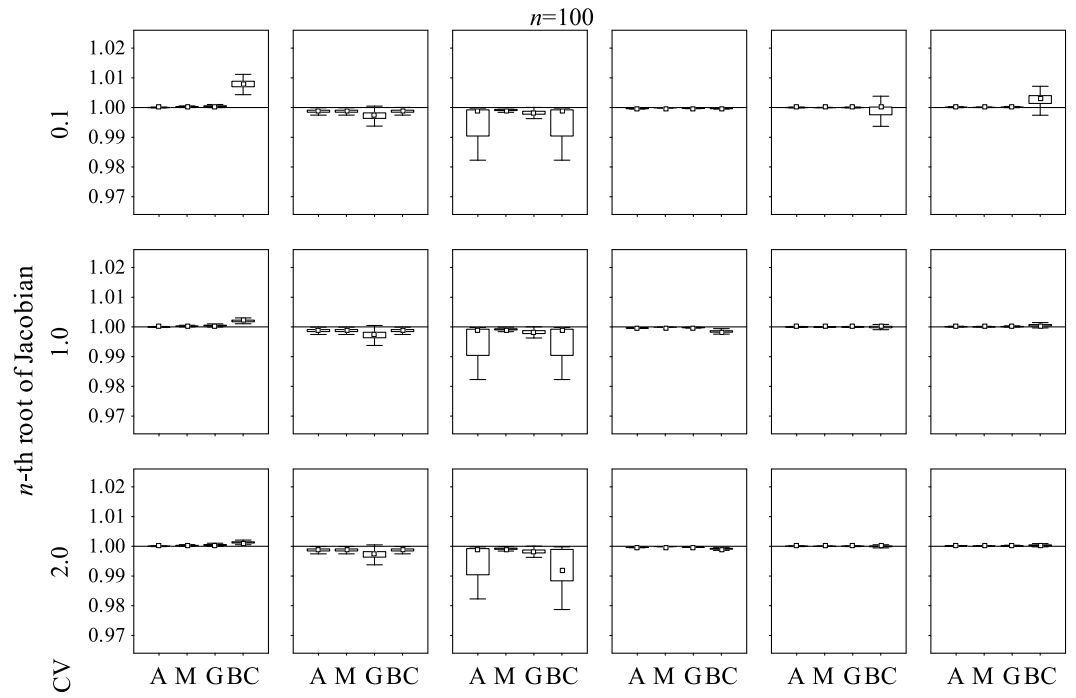

A M GBC

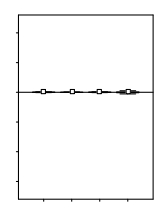

0.1

$$
\begin{gathered}
0.1379 \\
\text { Shape Parameter } \\
n=200
\end{gathered}
$$

Fig. 1: Box and whisker plot of the $n$-th root of Jacobian (A: $\phi_{S}$ with $x_{A}$, M: $\phi_{S}$ with $x_{M}$, G: $\phi_{S}$ with $x_{G}$, BC: $\left.\phi_{B C}\right)$ 
A Simple Modification of the Box and Cox Transformation
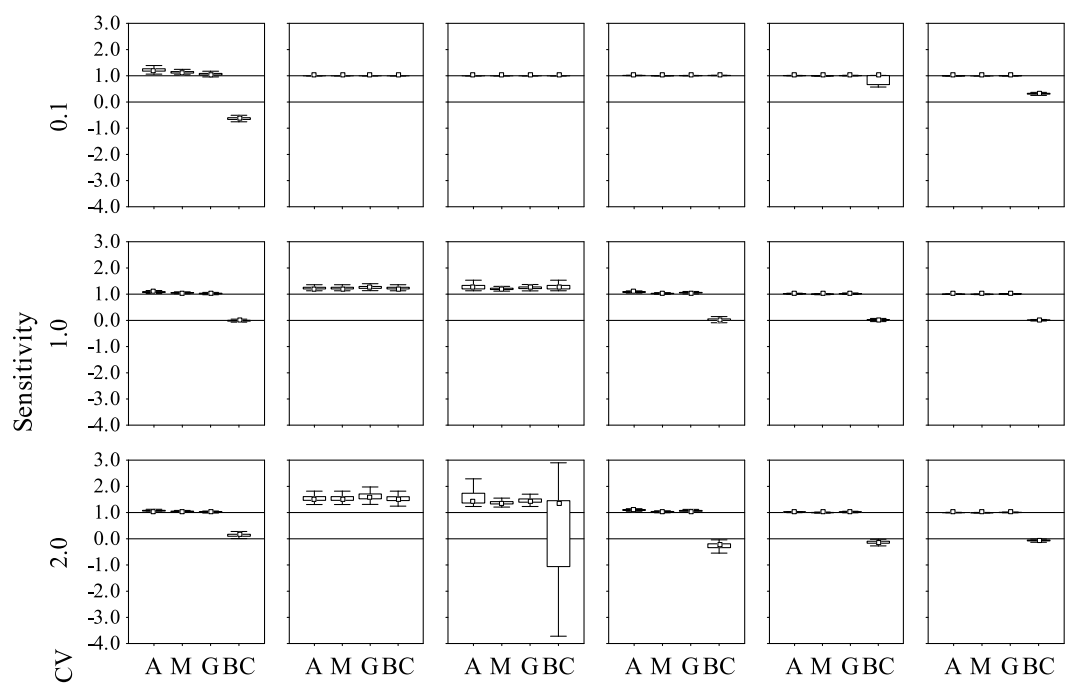

$-1.0$
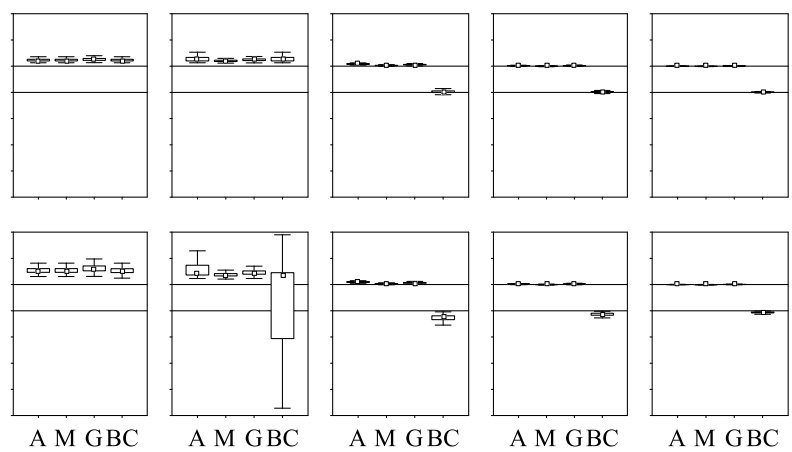

0.1

0.1379

0.6

1.0

Shape Parameter
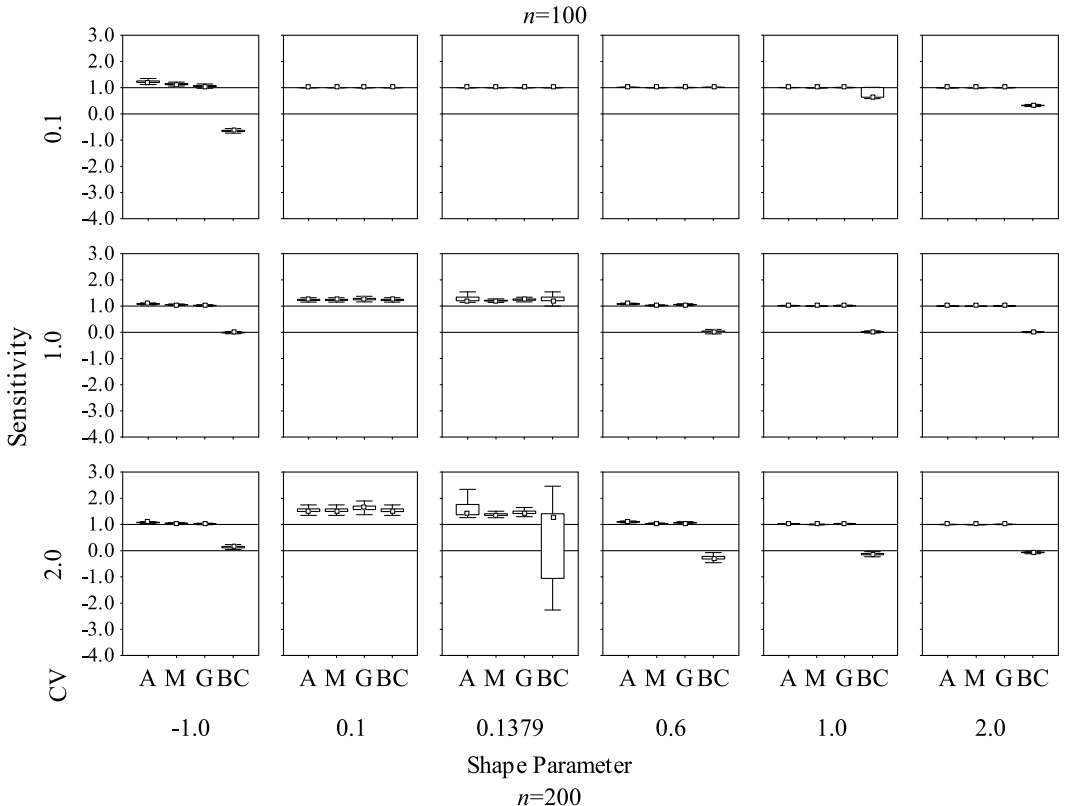

$-1.0$

0.1

1.0

2.0

Fig. 2: Box and whisker plot of the sensitivity of mean estimate (A: $\phi_{S}$ with $x_{A}, \mathrm{M}: \phi_{S}$ with $x_{M}, \mathrm{G}: \phi_{S}$ with $\left.x_{G}, \mathrm{BC}: \phi_{B C}\right)$ 

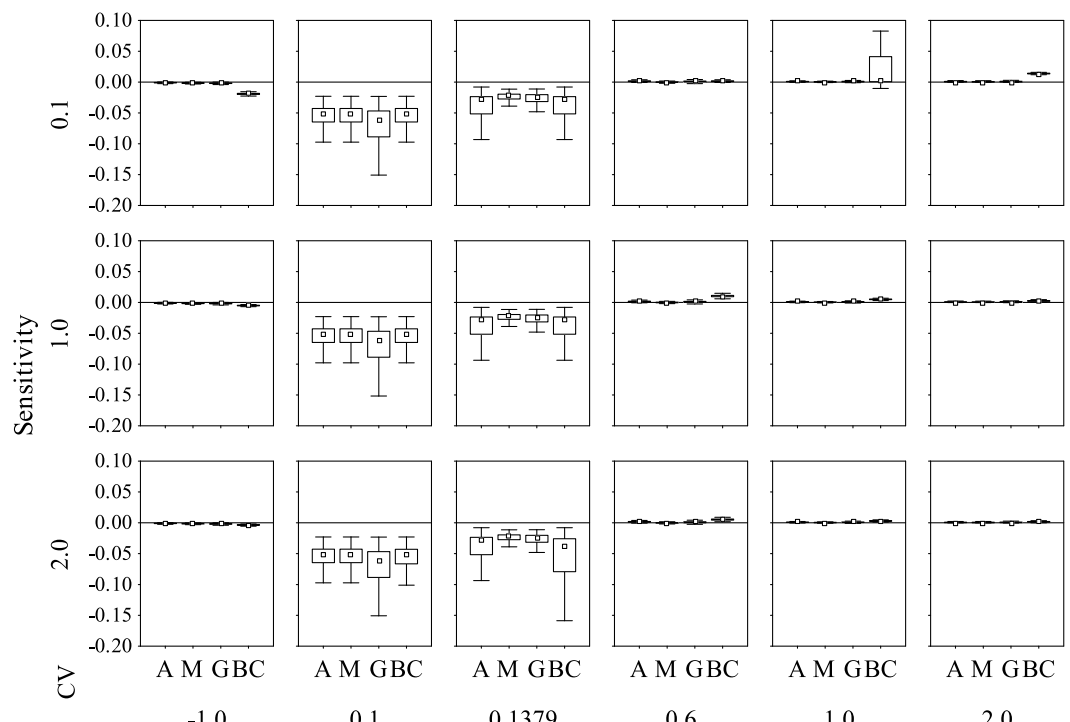

0.1

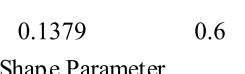

A M GBC

A M GBC

Shape Parameter
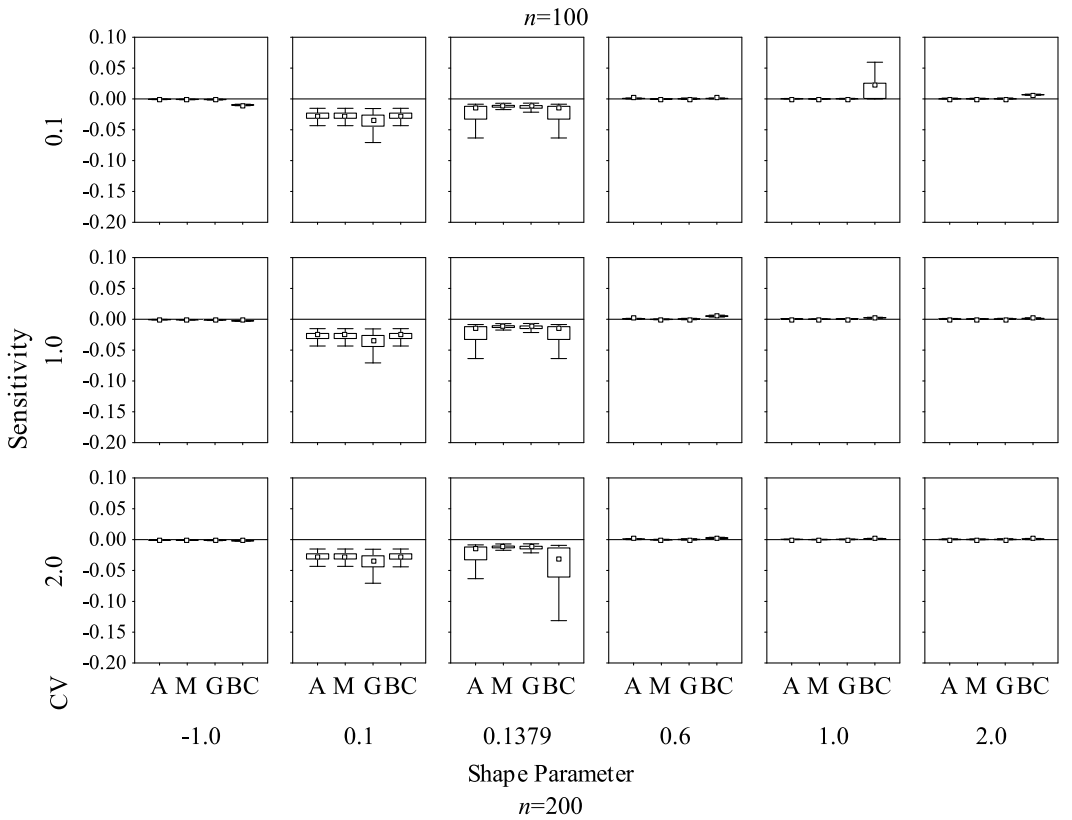

1.0

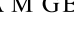

$n=200$

Fig. 3: Box and whisker plot of the sensitivity of variance estimate (A: $\phi_{S}$ with $x_{A}, \mathrm{M}$ : $\phi_{S}$ with $x_{M}, \mathrm{G}: \phi_{S}$ with $\left.x_{G}, \mathrm{BC}: \phi_{B C}\right)$ 
variance. Furthermore, the result from the simulation study shows that the normalized transformation provides more reliable estimates of the mean and variance. Thus, the median or geometric mean may be recommended as the constant in the scale of measurement unit. However, when the truncation of the distribution is not small enough to be ignored, we must be careful with the choice of the constant. These straightforwardly suggest that, in regression analysis, use of the normalized invariant transformation may provide more stable and reliable estimates of regression parameters, especially a constant term, and variance.

\section{Acknowledgements}

The authors are grateful to two referees for their constructive comments and helpful suggestions. This research was financially supported by a program grant from The Ministry of Education, Culture, Sports, Science and Technology (Grant-in-Aid for Scientific Research (C), NO.20500255).

\section{REFERENCES}

Akima, H. (1970). A new method of interpolation and smooth curve fitting based on local procedures. Journal of the Association for Computing Machinery 17, 589-602.

Box, G.E.P. and Cox, D.R. (1964). An analysis of transformations (with discussion). Journal of the Royal Statistical Society, Series B 26, 211-246.

Box, G.E.P. and Cox, D.R. (1982). An analysis of transformations revisited, rebutted. Journal of the American Statistical Association 77, 209-210.

Bickel, P.J. and Doksum, K.A. (1981). An analysis of transformations revisited. Journal of the American Statistical Association 76, 296-311.

Duan, N. (1993). Sensitivity analysis of Box-Cox power-transformation model: Contrast parameter. Biometrika $\mathbf{8 0}, 885-897$.

Goto, M., Matsubara, Y. and Tsuchiya, Y. (1983). Power-normal distribution and its applications. Reports of Statistical Application Research, JUSE 30(3), 8-28.

Guerra, V. O., Tapia, R.A. and Thompson, J.R. (1976). A random number generator for continuous random variables based on an interpolation procedure of Akima. In Proceedings of the Ninth Interface Symposium on Computer Science and Statistics, D.C. Hoaglin and R.E. Welsch (Eds.), 228-230, Boston: Prindle, Weber \& Schmidt,

Hamasaki, T. and Goto, M. (1996). Normalizing power transformations to scale invariance. Bulletin of the Japanese Society of Computational Statistics 9(1), 33-55. (in Japanese)

Hamasaki, T., Isomura, T., Ohtaki, M. and Goto, M. (1999). The Box and Cox powertransformation and its modifications. Japanese Journal of Applied Statistics 28, 179-190. (in Japanese)

Hernandez, J. and Johnson, R.A. (1980). The large-sample behavior of transformations to normality. Journal of the American Statistical Association 75, 855-869.

Hinkley, D.V. (1989). Modified profile likelihood in transformed linear models. Applied Statistics 38, 495-506.

Hinkley, D.V. and Runger, G. (1984). The analysis of transformed data. Journal of the American Statistical Association 79, 302-320.

Johnson, N.L., Kotz, S. and Balakrishnan, N. (1994). Continuous Univariate Distributions. 2nd Edition, New York: John Wiley \& Sons.

Sakia, R.M. (1992). The Box-Cox transformation technique: A review. The Statistician 41, $169-178$. 
HAMASAKI, SUGIMOTO and KIM

Schlesselman, J. (1971). Power-families: A note on the Box and Cox transformation. Journal of the Royal Statistical Society, Series B 33, 307-311.

(Received: May 6, 2010, Accepted: December 7, 2010) 